\title{
Erdős-Ko-Rado-Type Theorems for Colored Sets
}

\author{
Yu-Shuang Li and Jun Wang*† \\ Department of Applied Mathematics, Dalian University of Technology, \\ Dalian 116024, P. R. China \\ Submitted: Jun 7, 2006; Accepted: Dec 10, 2006; Published: Jan 3, 2007 \\ Mathematics Subject Classification: 05D05
}

\begin{abstract}
An Erdős-Ko-Rado-type theorem was established by Bollobás and Leader for $q$-signed sets and by $\mathrm{Ku}$ and Leader for partial permutations. In this paper, we establish an LYM-type inequality for partial permutations, and prove Ku and Leader's conjecture on maximal $k$-uniform intersecting families of partial permutations. Similar results on general colored sets are presented.
\end{abstract}

\section{Introduction}

Erdős, Ko and Rado proved in 1961 [10] that a family of pairwise intersecting $k$-subsets of an $n$-set cannot have more members than the family of $k$-subsets all of which contain a given element $a$, say, provided $k \leq\left\lfloor\frac{n}{2}\right\rfloor$. Bollobás in 1973 [3] established a stronger resultan LYM-type inequality, which says that if $\mathcal{A}$ is an intersecting antichain of subsets of an $n$-set, then $\sum_{k \geq 1} \frac{f_{k}}{\left(\begin{array}{c}n-1 \\ k-1\end{array}\right)} \leq 1$, where $f_{k}$ denotes the number of sets in $\mathcal{A}$ of size $k$ with $k \leq n / 2$. This inequality implies the Erdős-Ko-Rado Theorem. The original LYM inequality says that if $\mathcal{A}$ is an antichain of subsets of an $n$-set, then $\sum_{k=0}^{n} \frac{f_{k}}{\left(\begin{array}{c}n \\ k\end{array}\right)} \leq 1$, which yields a simple proof of Sperner's Theorem that $|\mathcal{A}|=\sum_{k=0}^{n} f_{k} \leq\left(\begin{array}{c}n \\ {\left[\frac{n}{2}\right]}\end{array}\right]$. This proof is due independently to Lubell, Yamamoto and Meschalkin, and therefore the inequality is known as the LYM-inequality (see [9] for detail).

In 1972 Katona presented a rather simple proof of the Erdős-Ko-Rado Theorem. By his technique one can usually establish an LYM-type inequality. By employing Katona's technique, in 1997, Bollobás and Leader [4] presented an Erdős-Ko-Rado theorem for $q$ signed sets where $q \geq 2$. A $q$-signed $k$-set is a pair $(A, f)$, where $A \subseteq[n]$ is a $k$-set and $f$

${ }^{*}$ Correspondence author, E-mail address: junwang@dlut.edu.cn

${ }^{\dagger}$ Supported by the National Natural Science Foundation of China (grant number: 10471016).

The EleCtronic Journal of COMBinatorics 14 (2007), \#R1 
is a function from $A$ to $[q]$. A family $\mathcal{F}$ of $q$-signed $k$-sets is intersecting if for any $(A, f)$, $(B, g) \in \mathcal{F}$ there exists $x \in A \cap B$ such that $f(x)=g(x)$.

Theorem 1.1 (Bollobás and Leader) Fix a positive integer $k \leq n$, and let $\mathcal{F}$ be an intersecting family of $q$-signed $k$-sets on $[n]$, where $q \geq 2$. Then $|\mathcal{F}| \leq\left(\begin{array}{l}n-1 \\ k-1\end{array}\right) q^{k-1}$. Unless $q=2$ and $k=n$, equality holds if and only if $\mathcal{F}$ consists of all q-signed $k$-sets $(A, f)$ such that $x_{0} \in A$ and $f\left(x_{0}\right)=\varepsilon_{0}$ for some fixed $x_{0} \in[n], \varepsilon_{0} \in[q]$.

Note that a $q$-signed set can be reformulated as an element of a generalized Boolean algebra. Let $M_{1}, M_{2}, \ldots, M_{n}$ be $n$ pairwise disjoint sets of the same cardinality $q$, say $M_{i}=\left\{x_{i, 1}, \ldots, x_{i, q}\right\}, i=1, \ldots, n$. The associated generalized Boolean algebra is defined to be the family

$$
\mathcal{B}(n, q)=\left\{C \subseteq M_{1} \cup M_{2} \cup \cdots \cup M_{n}:\left|C \cap M_{i}\right| \leq 1, i=1, \ldots, n\right\}
$$

ordered by containment. Given a $k$-set $C \in \mathcal{B}(n, q)$, say $C=\left\{x_{i_{1}, j_{1}}, \ldots, x_{i_{k}, j_{k}}\right\}$, we define a unique $q$-signed $k$-set $(A, f)$, where $A=\left\{i_{1}, \ldots, i_{k}\right\}$ and $f\left(i_{t}\right)=j_{t}$ for $t=1, \ldots, k$. It is evident that two sets in $\mathcal{B}(n, q)$ are intersecting if and only if the $q$-signed sets corresponding to them are intersecting. Deza and Frankl in 1983 [6] proved that if $\mathcal{F}$ is a $k$ uniform intersecting family in $\mathcal{B}(n, q)$, then $|\mathcal{F}| \leq\left(\begin{array}{l}n-1 \\ k-1\end{array}\right) q^{k-1}$ for $q \geq 2$ and $k=1,2, \ldots, n$, which is equivalent to the first part of Theorem 1.1. Engel [8] strengthened the result of Deza and Frankl to an LYM-type inequality as follows.

Theorem 1.2 (Engel) Assume $q \geq 2$ and let $\mathcal{F} \subseteq \mathcal{B}(n, q)$ be an intersecting antichain with profile $\left(a_{1}, \ldots, a_{n}\right)$, where $a_{k}=|\{A \in \mathcal{F}:|A|=k\}|$. Then

$$
\sum_{k=1}^{n} \frac{a_{k}}{\left(\begin{array}{l}
n-1 \\
k-1
\end{array}\right) q^{k-1}} \leq 1 .
$$

Note that when $\mathcal{F}$ is $k$-uniform, the inequality above implies $|\mathcal{F}|=a_{k} \leq\left(\begin{array}{c}n-1 \\ k-1\end{array}\right) q^{k-1}$. Note also that Erdős, Faigle and Kern in 1992 [11] gave a group-theoretic proof of Theorem 1.2 .

Recently, $\mathrm{Ku}$ and Leader [15] established an Erdős-Ko-Rado-type theorem for partial permutations. A $k$-partial permutation of $[n]$ is a pair $(A, f)$ where $A \subseteq[n]$ with $|A|=k$ and $f: A \rightarrow[n]$ is an injective map. Note that an $n$-partial permutation of $[n]$ is just a permutation on $[n]$. By $S_{n}$ we denote the set of all permutations on $[n]$. The intersecting property for partial permutations is defined in the same way as for signed sets, that is, a family $\mathcal{F}$ of partial permutations is intersecting if for any $(A, f),(B, g) \in \mathcal{F}$ there exists $x \in A \cap B$ such that $f(x)=g(x)$.

Theorem 1.3 (Ku and Leader) Fix $k, n$ with $k \leq n-1$ and let $\mathcal{F}$ be an intersecting family of $k$-partial permutations. Then

$$
|\mathcal{F}| \leq\left(\begin{array}{l}
n-1 \\
k-1
\end{array}\right) \frac{(n-1) !}{(n-k) !}
$$


They also showed that for $8 \leq k \leq n-3$, equality holds if and only if $\mathcal{F}$ consists of all $k$-partial permutations $(A, f)$ such that $x_{0} \in A$ and $f\left(x_{0}\right)=\varepsilon_{0}$ for some fixed $x_{0}, \varepsilon_{0} \in[n]$. And, they conjectured the following.

Conjecture 1.4 (Ku and Leader) Equality in Theorem 1.3 holds if and only if $\mathcal{F}$ consists of all $k$-partial permutations $(A, f)$ such that $x_{0} \in A$ and $f\left(x_{0}\right)=\varepsilon_{0}$ for some fixed $x_{0}, \varepsilon_{0} \in[n]$.

In fact, Theorem 1.3 and Conjecture 1.4 hold for $k=n$.

Theorem 1.5 Let $\mathcal{F}$ be an intersecting family in $S_{n}$. Then

(i) (Deza and Frankl [7]) $|\mathcal{F}| \leq(n-1)$ !.

(ii) (Cameron and Ku [5]) Equality in (i) holds if and only if $\mathcal{F}$ is a coset of the stabilizer of a point.

The result in (ii) was also deduced from a more general result on certain vertex transitive graphs in Larose and Malvenuto's paper [16].

Combining the signed sets and the partial permutations, we introduce the following concepts.

Let $N$ be a fixed finite set, and let $\mathfrak{p}_{n}$ be a subset of $N^{[n]}$, the set of all maps from $[n]$ to $N$. Then $\mathfrak{p}_{n}$ can be regarded as a set of colorings of $[n]$. Define

$$
\mathcal{B}\left(\mathfrak{p}_{n}\right)=\left\{\left(A,\left.f\right|_{A}\right): A \subseteq[n], f \in \mathfrak{p}_{n}\right\},
$$

where $\left.f\right|_{A}$ is the restriction of $f$ on $A$. We simply write the pair $\left(A,\left.f\right|_{A}\right)$ as $(A, f)$ for short, which will not cause confusions. Define an ordering on $\mathcal{B}\left(\mathfrak{p}_{n}\right)$ as follows:

$$
(A, f) \leq(B, g) \Leftrightarrow A \subseteq B \text { and }\left.g\right|_{A}=\left.f\right|_{A}
$$

With this ordering $\mathcal{B}\left(\mathfrak{p}_{n}\right)$ forms a ranked poset with the rank function $\rho(A, f)=|A|$. By $\mathcal{B}_{k}\left(\mathfrak{p}_{n}\right)$ we denote the set of all elements of rank $k$. An element of rank 1 is usually called an atom. An antichain of $\mathcal{B}\left(\mathfrak{p}_{n}\right)$ is a subset of which no two elements are comparable in $\mathcal{B}\left(\mathfrak{p}_{n}\right)$. For example, $\mathcal{B}_{k}\left(\mathfrak{p}_{n}\right)$ is an antichain.

From the definition, we see that $\mathcal{B}\left(\mathfrak{p}_{n}\right)$ is determined by the set of colorings $\mathfrak{p}_{n}$. If $\mathfrak{p}_{n}$ is the empty set, then $\mathcal{B}\left(\mathfrak{p}_{n}\right)$ is the boolean algebra $B_{n}$. Let $\mathfrak{q}_{n}=[q]^{[n]}$ for a positive integer $q \geq 2$, and let $\mathfrak{s}_{n}=S_{n}$. Then $\mathcal{B}\left(\mathfrak{q}_{n}\right)$ is the set of all $q$-signed sets, and $\mathcal{B}\left(\mathfrak{s}_{n}\right)$ is the set of all partial permutations.

Given an $A \subseteq[n]$, let $\left[\mathfrak{p}_{n}\right]_{A}$ denote the set of all pairs $(A, f) \in \mathcal{B}\left(\mathfrak{p}_{n}\right)$. We say $\mathfrak{p}_{n}$ is regular if the cardinality of $\left[\mathfrak{p}_{n}\right]_{A}$ depends only on $|A|$.

In the sequel of this paper, all sets of colorings concerned are assumed regular, and by $\left[\mathfrak{p}_{n}\right]_{k}$ we denote the cardinality of $\left[\mathfrak{p}_{n}\right]_{A}$ with $|A|=k$. Thus

$$
\left|\mathcal{B}_{k}\left(\mathfrak{p}_{n}\right)\right|=\left(\begin{array}{l}
n \\
k
\end{array}\right)\left[\mathfrak{p}_{n}\right]_{k}
$$


It is easy to verify that the sets of colorings $\mathfrak{q}_{n}$ and $\mathfrak{s}_{n}$ are regular with $\left[\mathfrak{q}_{n}\right]_{k}=q^{k}$ and $\left[\mathfrak{s}_{n}\right]_{k}=\frac{n !}{(n-k) !}$.

A subset $\mathcal{F}$ of $\mathcal{B}\left(\mathfrak{p}_{n}\right)$ is called an intersecting family if for any $(A, f),(B, g) \in \mathcal{F}$, there exists $x \in A \cap B$ such that $f(x)=g(x)$, in other words, both $(A, f)$ and $(B, g)$ are greater than the atom $\left(\{x\}, f_{0}\right)$ where $f_{0}$ is defined by $f_{0}(x)=f(x)=g(x)$. The profile $\left(a_{1}, a_{2}, \ldots\right)$ of $\mathcal{F}$ is given by $a_{k}=|\{(A, f) \in \mathcal{F}:|A|=k\}|$ for $k=1,2, \ldots, n$. We say $\mathcal{F}$ is $k$-uniform if $\mathcal{F} \subseteq \mathcal{B}_{k}\left(\mathfrak{p}_{n}\right)$. Let $\alpha$ be an atom of $\mathcal{B}\left(\mathfrak{p}_{n}\right)$, and set $\mathcal{S}_{k}(\alpha)=\left\{(A, f) \in \mathcal{B}_{k}\left(\mathfrak{p}_{n}\right):(A, f) \geq \alpha\right\}$. Then $\mathcal{S}_{k}(\alpha)$ is a $k$-uniform intersecting family, called a $k$-star. The regularity of $\mathfrak{p}_{n}$ implies that $\left|\mathcal{S}_{k}(\alpha)\right|=\left(\begin{array}{c}n-1 \\ k-1\end{array}\right)\left[\mathfrak{p}_{n-1}\right]_{k-1}$ for each atom $\alpha$.

For $1 \leq k \leq n$, we say $\mathcal{B}\left(\mathfrak{p}_{n}\right)$ has the EKR property for rank $k$ if every $k$-uniform intersecting family $\mathcal{F}$ satisfies $|\mathcal{F}| \leq\left(\begin{array}{l}n-1 \\ k-1\end{array}\right)\left[\mathfrak{p}_{n-1}\right]_{k-1}$. And, we say $\mathcal{B}\left(\mathfrak{p}_{n}\right)$ has the uniqueness property for rank $k$ if equality holds if and only if $\mathcal{F}$ is a $k$-star. We say $\mathcal{B}\left(\mathfrak{p}_{n}\right)$ satisfies an LYM-type inequality for rank $k$ if for each intersecting antichain $\mathcal{F}$ with profile $\left(a_{1}, a_{2}, \ldots, a_{k}\right)$, we have

$$
\sum_{i=1}^{k} \frac{a_{i}}{\left(\begin{array}{c}
n-1 \\
i-1
\end{array}\right)\left[\mathfrak{p}_{n-1}\right]_{i-1}} \leq 1 .
$$

(Note that the previous notions can be generalized to a ranked poset in a similar way.) Furthermore, we say $\mathcal{B}\left(\mathfrak{p}_{n}\right)$ has the local $E K R$ property for rank $k$ if for every $A \subseteq[n]$ with $|A|=k,\left[\mathfrak{p}_{n}\right]_{A}$ has the EKR property, that is, there is an $x_{0} \in A$ and a $y_{0} \in N$ such that $\left\{(A, f): f\left(x_{0}\right)=y_{0}\right\}$ is a maximum intersecting family in $\left[\mathfrak{p}_{n}\right]_{A}$.

Example 1.6 From Theorem 1.1 we see that $\mathcal{B}\left(\mathfrak{q}_{n}\right)$ has the EKR property for rank $n$. Recall that $\mathfrak{q}_{n}=[q]^{[n]}$, where $q$ is independent to $n$. We therefore obtain that $\mathcal{B}\left(\mathfrak{q}_{n}\right)$ has the local EKR property for all ranks $k=1,2, \ldots, n$. We believe that $\mathcal{B}\left(\mathfrak{s}_{n}\right)$ also has the local EKR property for every rank $k=1,2, \ldots, n$, but it can not follow from the EKR property for rank $n$, because the domain and the image of $\mathfrak{s}_{n}$ are dependent.

Remark 1.7 Generally, the local EKR property does not imply the EKR property. For example, when $\mathfrak{p}_{n}$ is empty, $\mathcal{B}\left(\mathfrak{p}_{n}\right)$ is the boolean algebra $B_{n}$. For every $A \subseteq[n]$ with $|A|>n / 2,\left[\mathfrak{p}_{n}\right]_{A}$ trivially has the EKR property, but $B_{n}$ has no the EKR property for ranks greater than $n / 2$.

In the next section, we first establish an LYM-type inequality for $\mathcal{B}\left(\mathfrak{s}_{n}\right)$ which deduces Theorem 1.3 immediately, then we prove Conjecture 1.4. Note that our proof of the conjecture does not depend on the LYM-type inequality, but only on the inequality in Theorem 1.3. In Section 3 we discuss the direct product of colorings (as sets), and present a theorem on its EKR property, an LYM-type inequality, and the uniqueness property. As a consequence, we give corresponding results on the direct product of $\mathfrak{q}_{n}$ and $\mathfrak{s}_{n}$.

\section{On partial permutations}

Recall that a partial permutation, as defined in [15], is a pair $(A, f)$, where $A \subseteq[n]$ and $f$ is an injection from $A$ into $[n]$. By our notation, $f \in \mathfrak{s}_{n}$, and $\mathcal{B}\left(\mathfrak{s}_{n}\right)$ denotes the set 
of all partial permutations. We first establish an LYM-type inequality for $\mathcal{B}\left(\mathfrak{s}_{n}\right)$. The techniques we use here are based on the ideas from $[4,13,15]$, which originally came from Katona [14].

As defined in [15], a cyclic ordering of $[n] \times[n]$ is a bijection $\sigma:[n] \times[n] \rightarrow\left[n^{2}\right]$. Given such cyclic ordering $\sigma$, we may arrange the elements of $[n] \times[n]$ on a cycle of length $n^{2}$ in the natural way. Let $k, n$ be positive integers where $k \leq n-1$. A $k$-interval in the cyclic ordering is a sequence of $k$ elements $\left(x_{1}, \varepsilon_{1}\right), \ldots,\left(x_{k}, \varepsilon_{k}\right)$ in $[n] \times[n]$ such that $\sigma\left(x_{i+1}, \varepsilon_{i+1}\right)=\sigma\left(x_{i}, \varepsilon_{i}\right)+1\left(\bmod n^{2}\right)$ for $1 \leq i \leq k-1$, and denote this $k$-interval by $\left[\left(x_{1}, \varepsilon_{1}\right), \ldots,\left(x_{k}, \varepsilon_{k}\right)\right]$. A $k$-partial permutation $(A, f)$ is compatible with a cyclic ordering $\sigma$, written as $(A, f) \prec \sigma$, if there is a $k$-interval $\left[\left(x_{1}, \varepsilon_{1}\right), \ldots,\left(x_{k}, \varepsilon_{k}\right)\right]$ in the ordering such that $x_{i} \in A$ and $f\left(x_{i}\right)=\varepsilon_{i}$ for $i=1,2, \ldots, k$.

The following $n !^{2}$ good cyclic orderings constructed by Ku and Leader in [15] play an essential role for our argument: the standard good cyclic ordering $\tau$ defined by $\tau(x, \varepsilon)=$ $x+d n$ where $d=\varepsilon-x(\bmod n)$, and other good cyclic orderings $\tau_{\pi \pi^{\prime}}$ defined by $\tau_{\pi \pi^{\prime}}(x, \varepsilon)=$ $\tau\left(\pi(x), \pi^{\prime}(\varepsilon)\right)$, where $\pi, \pi^{\prime} \in S_{n}$. Write the set of these good cyclic orderings as $\mathcal{C}_{n}$.

Lemma 2.1 Let $k \leq n-1$ be a positive integer. Then every $k$-partial permutation is exactly compatible with $n^{2} k !(n-k) !^{2}$ good cyclic orderings in $\mathcal{C}_{n}$.

Proof. Let $(A, f)$ be any selected $k$-partial permutation with $A=\left\{a_{1}, \ldots, a_{k}\right\}$ and $f(A)=\left\{b_{1}, \ldots, b_{k}\right\}$ where $b_{i}=f\left(a_{i}\right), i=1, \ldots, k$. Then, for a $\sigma \in \mathcal{C}_{n},(A, f)$ is compatible with $\sigma$ if and only if there is a $k$-interval of $\sigma$, say $\left[\left(x_{1}, \varepsilon_{1}\right), \ldots,\left(x_{k}, \varepsilon_{k}\right)\right]$, such that $\left\{\left(x_{1}, \varepsilon_{1}\right), \ldots,\left(x_{k}, \varepsilon_{k}\right)\right\}=\left\{\left(a_{1}, b_{1}\right), \ldots,\left(a_{k}, b_{k}\right)\right\}$, which says that if $\sigma=\tau_{\pi \pi^{\prime}}$, then there is a $k$-interval $\left[\left(y_{1}, \theta_{1}\right), \ldots,\left(y_{k}, \theta_{k}\right)\right]$ in $\tau$ such that

$$
\left\{\left(y_{1}, \theta_{1}\right), \ldots,\left(y_{k}, \theta_{k}\right)\right\}=\left\{\left(\pi\left(a_{1}\right), \pi^{\prime}\left(b_{1}\right)\right), \ldots,\left(\pi\left(a_{k}\right), \pi^{\prime}\left(b_{k}\right)\right)\right\}
$$

as two sets. Clearly, $\tau$ has $n^{2}$ many $k$-intervals, and for each one, there are $k !(n-k) !^{2}$ pairs $\left(\pi, \pi^{\prime}\right)$ 's satisfying $(2)$, completing the proof.

Theorem 2.2 Let $\mathcal{F}$ be an intersecting antichain of partial permutations with profile $\left(a_{1}, \ldots, a_{n-1}\right)$. Then

$$
\sum_{k=1}^{n-1} \frac{a_{k}}{\left(\begin{array}{l}
n-1 \\
k-1
\end{array}\right) \frac{(n-1) !}{(n-k) !}} \leq 1
$$

Proof. The argument below is standard, see e.g. [1, p.73]. For each $\sigma \in \mathcal{C}_{n}$ and each partial permutation $\left(A_{i}, f_{i}\right)$ in $\mathcal{F}$, define

$$
F\left(\sigma,\left(A_{i}, f_{i}\right)\right)=\left\{\begin{array}{cl}
\frac{1}{\left|A_{i}\right|}, & \text { if }\left(A_{i}, f_{i}\right) \prec \sigma ; \\
0, & \text { otherwise }
\end{array}\right.
$$

We count $\sum_{i, \sigma} F\left(\sigma,\left(A_{i}, f_{i}\right)\right)$ in two different ways. First we have

$$
\sum_{i, \sigma} F\left(\sigma,\left(A_{i}, f_{i}\right)\right)=\sum_{\sigma} \sum_{\left(A_{i}, f_{i}\right) \prec \sigma} \frac{1}{\left|A_{i}\right|} .
$$


Consider the inner sum where $\sigma$ is fixed. Choose $\left(A_{j}, f_{j}\right)$ from $\left(A_{i}, f_{i}\right)$ 's compatible with $\sigma$ such that $\rho\left(A_{j}, f_{j}\right)$ is the smallest of the $\rho\left(A_{i}, f_{i}\right)$. Clearly, there are at most $\left|A_{j}\right|$ of the intervals of $\sigma$ may intersect pairwise, i.e. at most $\left|A_{j}\right|$ terms in the inner sum, each $\leq \frac{1}{\left|A_{j}\right|}$. Therefore the inner sum is at most $\left|A_{j}\right| \cdot \frac{1}{\left|A_{j}\right|}=1$, and we have

$$
\sum_{i, \sigma} F\left(\sigma,\left(A_{i}, f_{i}\right)\right) \leq \sum_{\sigma} 1=n !^{2} .
$$

On the other hand, we have

$$
\sum_{i, \sigma} F\left(\sigma,\left(A_{i}, f_{i}\right)\right)=\sum_{i} \frac{1}{\left|A_{i}\right|} n^{2}\left|A_{i}\right| !\left(n-\left|A_{i}\right|\right) !^{2}=\sum_{k=1}^{n-1} a_{k} n^{2}(k-1) !(n-k) !^{2} .
$$

Comparing (3) and (4), we obtain the desired inequality.

From Theorem 2.2 it follows immediately that $|\mathcal{F}| \leq\left(\begin{array}{l}n-1 \\ k-1\end{array}\right) \frac{(n-1) !}{(n-k) !}$ if $\mathcal{F}$ is a $k$-uniform intersecting family. The theorem below confirms Conjecture 1.4.

Theorem 2.3 Fix $k$, $n$ with $k \leq n-1$. Suppose that $\mathcal{F}$ is a $k$-uniform intersecting family in $\mathcal{B}\left(\mathfrak{s}_{n}\right)$ with $|\mathcal{F}|=\left(\begin{array}{c}n-1 \\ k-1\end{array}\right) \frac{(n-1) !}{(n-k) !}$. Then $\mathcal{F}=S_{k}(\alpha)$ for some atom $\alpha \in \mathcal{B}_{1}\left(\mathfrak{s}_{n}\right)$.

Proof. From a key observation in the well-known argument of Katona [14] we know that given a $\sigma \in \mathcal{C}_{n}$, there are at most $k$ of the $k$-intervals of it may intersect pairwise, since $2 k<n^{2}$. Suppose $|\mathcal{F}|=\left(\begin{array}{c}n-1 \\ k-1\end{array}\right) \frac{(n-1) !}{(n-k) !}$. Then each $\sigma \in \mathcal{C}_{n}$ must contain exactly $k$ members of $\mathcal{F}$, and since the corresponding $k$-intervals must intersect pairwise, all these intervals must contain a fixed element of $[n] \times[n]$. We shall denote this fixed element (depending on $\mathcal{F}$ ) by $\left(x^{(\sigma)}, \varepsilon^{(\sigma)}\right)$, and call each $k$-interval containing $\left(x^{(\sigma)}, \varepsilon^{(\sigma)}\right)$ in $\sigma$ an $\mathcal{F}$-interval, which corresponds to an element of $\mathcal{F}$.

Consider the standard ordering $\tau$, and assume without loss of generality that $\left(x^{(\tau)}, \varepsilon^{(\tau)}\right)$ $=(n, n)$. Then, in $\tau$, the $(2 k-1)$-interval $[(n-k+1, n-k+1), \ldots,(n, n),(1,2),(2,3), \ldots$, $(k-1, k)$ ] contains $k \mathcal{F}$-intervals.

Let $\mathcal{C}_{n}^{\prime}$ denote the set of good cyclic orderings $\tau_{\pi \pi^{\prime}}$ 's with $\pi(n)=n$ and $\pi^{\prime}(n)=n$. We claim that $\left(x^{\left(\tau_{\pi \pi^{\prime}}\right)}, \varepsilon^{\left(\tau_{\pi \pi^{\prime}}\right)}\right)=(n, n)$ for any $\tau_{\pi \pi^{\prime}} \in \mathcal{C}_{n}^{\prime}$.

We first prove $\left(x^{\left(\tau_{\pi \pi}\right)}, \varepsilon^{\left(\tau_{\pi \pi}\right)}\right)=(n, n)$. Set $I=\{(i, i): 1 \leq i \leq n-1\}$ and $\bar{I}=[n] \times[n] \backslash$ $(I \cup\{(n, n)\})$. Then $(\pi \times \pi)(I)=\{(\pi(i), \pi(i)): 1 \leq i \leq n-1\}=I$ and $(\pi \times \pi)(\bar{I})=\bar{I}$. Suppose $\left(x^{\left(\tau_{\pi \pi}\right)}, \varepsilon^{\left(\tau_{\pi \pi}\right)}\right) \neq(n, n)$. Then $\left(x^{\left(\tau_{\pi \pi}\right)}, \varepsilon^{\left(\tau_{\pi \pi}\right)}\right) \in I$ or $\left(x^{\left(\tau_{\pi \pi}\right)}, \varepsilon^{\left(\tau_{\pi \pi}\right)}\right) \in \bar{I}$. If the former, then $\tau_{\pi \pi}$ has an $\mathcal{F}$-interval contained in $I$, which is clearly disjoint with the $\mathcal{F}$ interval $[(n, n),(1,2), \ldots,(k-1, k)]$; if the latter, then $\tau_{\pi \pi}$ has an $\mathcal{F}$-interval contained in $\bar{I}$, which is clearly disjoint with the $\mathcal{F}$-interval $[(n-k+1, n-k+1), \ldots,(n, n)]$. It yields contradictions in both cases.

Suppose now $\left(x^{\left(\tau_{\pi \pi^{\prime}}\right)}, \varepsilon^{\left(\tau_{\pi \pi^{\prime}}\right)}\right) \neq(n, n)$ for some $\tau_{\pi \pi^{\prime}} \in \mathcal{C}_{n}^{\prime}$ with $\pi \neq \pi^{\prime}$. Then $\tau_{\pi \pi^{\prime}}$ has an $\mathcal{F}$-interval, written as $J$, which contains no $(n, n)$. From the above discussion we see that $J \not \subset I$ and $J \not \subset \bar{I}$. Set $I \cap J=\left\{\left(a_{1}, a_{1}\right), \ldots,\left(a_{r}, a_{r}\right)\right\}$ where $1 \leq r<k$. Define a permutation $\pi$ by $\pi^{-1}(i)=a_{i}$ for $i \in[n]$ with $a_{n}=n$. Then $\tau_{\pi \pi} \in \mathcal{C}_{n}^{\prime}$, and $\tau_{\pi \pi}$ has an $\mathcal{F}$-interval 
which is contained in the $(n-1)$-interval $\left[\left(a_{r+1}, a_{r+1}\right), \ldots,(n, n),\left(a_{1}, a_{2}\right), \ldots,\left(a_{r-1}, a_{r}\right)\right]$. It is clear that $J$ is disjoint with this $(n-1)$-interval. It yields a contradiction again.

Therefore, we have $\left(x^{\left(\tau_{\pi \pi^{\prime}}\right)}, \varepsilon^{\left(\tau_{\pi \pi^{\prime}}\right)}\right)=\left(x^{(\tau)}, \varepsilon^{(\tau)}\right)=(n, n)$ for any $\tau_{\pi \pi^{\prime}} \in \mathcal{C}_{n}^{\prime}$. However, from Lemma 2.1 we know that if $(A, f)$ is any selected $k$-partial permutation with $n \in A$ and $f(n)=n$, then there are $k !(n-k) !^{2}$ pairs $\left(\pi, \pi^{\prime}\right)$ 's such that $\tau_{\pi \pi^{\prime}} \in \mathcal{C}_{n}^{\prime}$ and $(A, f) \prec \tau_{\pi \pi^{\prime}}$. It follows that $\mathcal{F}$ consists of all $k$-partial permutations $(A, f)$ with $n \in A$ and $f(n)=n$, as required.

\section{$3 \quad$ Direct product of colorings}

Let $\mathfrak{p}_{n}$ and $\mathfrak{p}_{n}^{\prime}$ be two sets of colorings. As two sets we consider their direct product $\mathfrak{p}_{n} \times \mathfrak{p}_{n}^{\prime}$, whose element $(f, g)$ is regarded as a function on $[n]$. We thus get a new set of colorings from the old ones, and write $\mathcal{B}\left(\mathfrak{p}_{n} \times \mathfrak{p}_{n}^{\prime}\right)=\left\{(A, f, g): A \subseteq[n], f \in \mathfrak{p}_{n}, g \in \mathfrak{p}_{n}^{\prime}\right\}$. From definition it is easy to see that $\mathcal{B}\left(\mathfrak{p}_{n} \times \mathfrak{p}_{n}^{\prime}\right)$ and $\mathcal{B}\left(\mathfrak{p}_{n}^{\prime} \times \mathfrak{p}_{n}\right)$ are isomorphic; $\mathfrak{p}_{n} \times \mathfrak{p}_{n}^{\prime}$ is regular if both $\mathfrak{p}_{n}$ and $\mathfrak{p}_{n}^{\prime}$ are regular, and $\left[\mathfrak{p}_{n} \times \mathfrak{p}_{n}^{\prime}\right]_{k}=\left[\mathfrak{p}_{n}\right]_{k}\left[\mathfrak{p}_{n}^{\prime}\right]_{k}$ for $1 \leq k \leq n$. More generally, we may consider the product $\mathfrak{p}_{n}^{(1)} \times \cdots \times \mathfrak{p}_{n}^{(m)}$ and write an element of $\mathcal{B}\left(\mathfrak{p}_{n}^{(1)} \times \cdots \times \mathfrak{p}_{n}^{(m)}\right)$ as $\left(A, f_{1}, \ldots, f_{m}\right)$ where $A \subseteq[n]$ and $f_{i} \in \mathfrak{p}_{n}^{(i)}$ for $i=1, \ldots, m$.

We may reformulate $\left(A, f_{1}, \ldots, f_{m}\right)$ as a matrix $\left[\alpha_{1}, \ldots, \alpha_{n}\right]$, where $\alpha_{i}=\left(a_{1 i}, \ldots, a_{m i}\right)^{T}$ is a column vector defined by

$$
a_{j i}=\left\{\begin{array}{cc}
f_{j}(i) & \text { if } i \in A, \\
0 & \text { if } i \notin A,
\end{array} \text { for } j=1,2, \ldots, m\right.
$$

The rank of $\left[\alpha_{1}, \ldots, \alpha_{n}\right]$ is given by the number of nonzero $\alpha_{i}$ 's. Let $M\left(\mathfrak{p}_{n}^{(1)} \times \cdots \times \mathfrak{p}_{n}^{(m)}\right)$ denote the set of all such matrices. An order relation on $M\left(\mathfrak{p}_{n}^{(1)} \times \cdots \times \mathfrak{p}_{n}^{(m)}\right)$ is defined by

$$
\left[\alpha_{1}, \ldots, \alpha_{n}\right] \leq\left[\beta_{1}, \ldots, \beta_{n}\right] \text { iff } \alpha_{i}=0 \text { (vector) or } \alpha_{i}=\beta_{i} \text { for } i=1,2, \ldots, n \text {. }
$$

Then, as posets, $M\left(\mathfrak{p}_{n}^{(1)} \times \cdots \times \mathfrak{p}_{n}^{(m)}\right)$ is isomorphic to $\mathcal{B}\left(\mathfrak{p}_{n}^{(1)} \times \cdots \times \mathfrak{p}_{n}^{(m)}\right)$, so they both can be regarded as generalizations of the function lattice (see [2] and [12]).

Theorem 3.1 Let $\mathfrak{p}_{n}$ and $\mathfrak{p}_{n}^{\prime}$ be two sets of regular colorings, and let $k$ be a positive integer with $1 \leq k \leq n$.

(i) If both $\mathcal{B}\left(\mathfrak{p}_{n}\right)$ and $\mathcal{B}\left(\mathfrak{p}_{n}^{\prime}\right)$ have the EKR property for rank $k$ and one of them has the local EKR property for rank $k$, then $\mathcal{B}\left(\mathfrak{p}_{n} \times \mathfrak{p}_{n}^{\prime}\right)$ also has the EKR property for rank $k$;

(ii) If both $\mathcal{B}\left(\mathfrak{p}_{n}\right)$ and $\mathcal{B}\left(\mathfrak{p}_{n}^{\prime}\right)$ have the uniqueness property for rank $k$, then $\mathcal{B}\left(\mathfrak{p}_{n} \times \mathfrak{p}_{n}^{\prime}\right)$ also has the uniqueness property for rank $k$;

(iii) If $\mathcal{B}\left(\mathfrak{p}_{n}\right)$ satisfies an LYM-type inequality for rank $k$, and $\mathcal{B}\left(\mathfrak{p}_{n}^{\prime}\right)$ has the local EKR properties for ranks from 1 to $k$, then $\mathcal{B}\left(\mathfrak{p}_{n} \times \mathfrak{p}_{n}^{\prime}\right)$ satisfies an LYM-type inequality for rank $k$. 
Proof. (i) Let $\mathcal{F}$ be a $k$-uniform intersecting family in $\mathcal{B}\left(\mathfrak{p}_{n} \times \mathfrak{p}_{n}^{\prime}\right)$. Put

$$
\mathcal{F}_{1}=\left\{(A, f): \text { there is a } g \in \mathfrak{p}_{n}^{\prime} \text { such that }(A, f, g) \in \mathcal{F}\right\}
$$

and

$$
\mathcal{F}_{2}=\left\{(A, g): \text { there is a } f \in \mathfrak{p}_{n} \text { such that }(A, f, g) \in \mathcal{F}\right\} .
$$

Then $\mathcal{F}_{1}$ and $\mathcal{F}_{2}$ are $k$-uniform intersecting families in $\mathcal{B}\left(\mathfrak{p}_{n}\right)$ and $\mathcal{B}\left(\mathfrak{p}_{n}^{\prime}\right)$, respectively, yielding $\left|\mathcal{F}_{1}\right| \leq\left(\begin{array}{c}n-1 \\ k-1\end{array}\right)\left[\mathfrak{p}_{n-1}\right]_{k-1}$ and $\left|\mathcal{F}_{2}\right| \leq\left(\begin{array}{c}n-1 \\ k-1\end{array}\right)\left[\mathfrak{p}_{n-1}^{\prime}\right]_{k-1}$. Now, suppose that $\mathcal{B}\left(\mathfrak{p}_{n}^{\prime}\right)$ has the local EKR property for rank $k$. Then, for each $(A, f) \in \mathcal{F}_{1}$, there are at most $\left[\mathfrak{p}_{n-1}^{\prime}\right]_{k-1}$ many $g \in \mathfrak{p}_{n}^{\prime}$ such that $(A, f, g) \in \mathcal{F}$, which implies

$$
|\mathcal{F}| \leq\left(\begin{array}{l}
n-1 \\
k-1
\end{array}\right)\left[\mathfrak{p}_{n-1}\right]_{k-1}\left[\mathfrak{p}_{n-1}^{\prime}\right]_{k-1}=\left(\begin{array}{c}
n-1 \\
k-1
\end{array}\right)\left[\mathfrak{p}_{n-1} \times \mathfrak{p}_{n-1}^{\prime}\right]_{k-1},
$$

as desired.

(ii) Suppose that $\mathcal{F}$ is a maximum $k$-uniform intersecting family in $\mathcal{B}\left(\mathfrak{p}_{n} \times \mathfrak{p}_{n}^{\prime}\right)$, that is, equality in (7) holds. This implies that $\left|\mathcal{F}_{1}\right|=\left(\begin{array}{c}n-1 \\ k-1\end{array}\right)\left[\mathfrak{p}_{n-1}\right]_{k-1}$ and $\left|\mathcal{F}_{2}\right|=\left(\begin{array}{c}n-1 \\ k-1\end{array}\right)\left[\mathfrak{p}_{n-1}^{\prime}\right]_{k-1}$, so $\mathcal{F}_{i}$ is a star, written as $S_{k}\left(\alpha_{i}\right)$, where $i=1,2$. Put $\alpha_{1}=\left(\left\{x_{0}\right\}, f_{0}\right) \in \mathcal{B}_{1}\left(\mathfrak{p}_{n}\right)$ and $\alpha_{2}=\left(\left\{y_{0}\right\}, g_{0}\right) \in \mathcal{B}_{1}\left(\mathfrak{p}_{n}^{\prime}\right)$. A careful analysis of the situation shows that $x_{0}=y_{0}$ and $\mathcal{F}=S_{k}(\alpha)$ where $\alpha=\left(\left\{x_{0}\right\}, f_{0}, g_{0}\right)$, as desired.

(iii) Let $\mathcal{F}$ be an intersecting antichain in $\mathcal{B}\left(\mathfrak{p}_{n} \times \mathfrak{p}_{n}^{\prime}\right)$ with profile $\left(a_{1}, a_{2}, \ldots, a_{k}\right)$, let $\mathcal{F}_{1}$ be as defined in (5) with profile $\left(b_{1}, b_{2}, \ldots, b_{k}\right)$, and let $\mathcal{F}_{2}$ be as defined in (6). Since $\mathcal{B}\left(\mathfrak{p}_{n}^{\prime}\right)$ has the local EKR property from rank 1 to rank $k$, we have that $a_{i} \leq b_{i}\left[\mathfrak{p}_{n-1}^{\prime}\right]_{i-1}$ for $i=1,2, \ldots, k$, so

$$
\begin{aligned}
\sum_{i=1}^{k} \frac{a_{i}}{\left(\begin{array}{c}
n-1 \\
i-1
\end{array}\right)\left[\mathfrak{p}_{n-1} \times \mathfrak{p}_{n-1}^{\prime}\right]_{i-1}} & \leq \sum_{i=1}^{k} \frac{b_{i}\left[\mathfrak{p}_{n-1}^{\prime}\right]_{i-1}}{\left(\begin{array}{c}
n-1 \\
i-1
\end{array}\right)\left[\mathfrak{p}_{n-1}\right]_{i-1}\left[\mathfrak{p}_{n-1}^{\prime}\right]_{i-1}} \\
& =\sum_{i=1}^{k} \frac{b_{i}}{\left(\begin{array}{c}
n-1 \\
i-1
\end{array}\right)\left[\mathfrak{p}_{n-1}\right]_{i-1}} \leq 1,
\end{aligned}
$$

as desired.

As an application we consider $\mathcal{B}\left(\mathfrak{q}_{n} \times \mathfrak{s}_{n}\right)$. We have known that for each $k \leq n-1$, both $\mathcal{B}\left(\mathfrak{q}_{n}\right)$ and $\mathcal{B}\left(\mathfrak{s}_{n}\right)$ have the EKR property for rank $k$, the uniqueness property for rank $k$, and satisfies an LYM-type inequality for $\operatorname{rank} k, \mathcal{B}\left(\mathfrak{q}_{n}\right)$ also has the local EKR property for rank $k$. From Theorem 3.1 we immediately obtain the following

Corollary 3.2 Let $\mathcal{F}$ be an intersecting antichain in $\mathcal{B}\left(\mathfrak{q}_{n} \times \mathfrak{s}_{n}\right)$ with profile $\left(a_{1}, \ldots, a_{n-1}\right)$. Then

$$
\sum_{k=1}^{n-1} \frac{a_{k}}{\left(\begin{array}{c}
n-1 \\
k-1
\end{array}\right) \frac{(n-1) !}{(n-k) !} q^{k-1}} \leq 1 .
$$

Equality holds if and only if there is a $k$ with $1 \leq k \leq n-1$ such that $\mathcal{F}$ is $k$-uniform and $\mathcal{F}$ is a k-star. 


\section{Acknowledgements}

The authors wish to thank Hong Feng for her valuable discussions. The authors are also grateful to the anonymous referee for carefully reading the manuscript and many improved suggestions and corrections.

\section{References}

[1] I. Anderson, Combinatorics of Finite sets, Oxford University Press, Oxford, 1987.

[2] C. Bey, The Erdős-Ko-Rado bound for the function lattice, Discrete Appl. Math., 95 (1999), 115-125.

[3] B. Bollobás, Sperner systems consisting of pairs of complementary subsets, J. Combin. Theory Ser. A, 15 (1973), 363-366.

[4] B. Bollobás and I. Leader, An Erdős-Ko-Rado theorem for signed sets, Comput. Math. Appl., 34 (1997), 9-13.

[5] P. J. Cameron and C. Y. Ku, Intersecting families of permutations, European J. Combin., 24 (2003), 881-890.

[6] M. Deza and P. Frankl, Erdős-Ko-Rado theorem-22 years later, SIAM J. Alg. Disc. Methods, 4 (1983), 419-431.

[7] M. Deza and P. Frankl, On the maximum number of permutations with given maximal or minimal distance, J. Combin. Theory Ser. A, 22 (1977), 352-360.

[8] K. Engel, An Erdős-Ko-Rado theorem for the subcubes of a cube, Combinatorica, 4 (1984), 133-140.

[9] K. Engel, Sperner Theory, Cambridge University Press, Cambridge, 1997.

[10] P. Erdős, C. Ko and R. Rado, Intersection theorems for systems of finite sets, Quart. J. Math. Oxford Ser., 2 (1961), 313-318.

[11] P. L. Erdős, U. Faigle and W. Kern, A group-theoretic setting for some intersecting Sperner families, Combin. Probab. Comput., 1 (1992), 323-334.

[12] P. L. Erdős, A. Seress, and L. A. Székely, Non-trivial $t$-intersection in the function lattice, Ann. Comb., 9 (2005), 177-187.

[13] C. Greene, G. Katona and D. J. Kleitman, Extensions of the Erdős-Ko-Rado theorem, Studies in Appl. Math., 55 (1976), 1-8.

[14] G. O. H. Katona, A simple proof of the Erdős-Ko-Rado theorem, J. Combin. Theory Ser. B, 13 (1972), 183-184.

[15] C. Y. Ku and I. Leader, An Erdős-Ko-Rado theorem for partial permutations, Discrete Math., 306 (2006), 74-86.

[16] B. Larose and C. Malvenuto, Stable sets of maximal size in Kneser-type graphs, European J. Combin., 25 (2004), 657-673. 